\section{Near-Field Acoustic Microscopy}

\author{
S. Hirsekorn, U. Rabe, W. Arnold \\ Fraunhofer Institut für zerstörungsfreie Prüfverfahren (IzfP), Saarbrücken
}

\begin{abstract}
Analysis shows that acoustic imaging based on the detection of ultrasonic fields using a modified atomic force microscope operated in the near-field mode not only offers nanoscale resolution but also opens the way forward to elastic imaging.
\end{abstract}

Conventional high-resolution acoustic imaging techniques offer important tools for materials evaluation and nondestructive testing. For instance, they allow elastic properties and defect distributions to be determined with a spatial resolution that depends of the wavelength of the imaging ultrasound. Research over the last few years has shown that acoustic images with a lateral resolution comparable to the tip diameter can be obtained using an atomic force microscope (AFM; see Fig. 1).

In a low-frequency set-up, a 10-50 $\mu \mathrm{m}$ in radius sensor tip is vibrated at a frequency of typically $100 \mathrm{kHz}$ while in contact with the sample surface [1]. The periodic impacts on the sample's surface generate an acoustic wave which is detected with a piezoelectric transducer. Reversed operation, i.e., sound generation with an ultrasonic transducer in contact with the sample and detection using a scanning force microscope has also been demonstrated [2].

Ultrasonic vibration in the $\mathrm{MHz}$ frequency range can also be detected by AFMs, either directly using a mixing technique [3] or indirectly using the mean deflection of the cantilever used to support the sensor. This deflection reflects the nonlinear force between the tip and surface of the sample surface [4-7].

Forced ultrasonic vibrations in an AFM's cantilever measured using a highspeed detector give a direct measurement of ultrasonic vibrations in the sample [ 7 , 8]. Furthermore, if a low-frequency lateral vibration is superimposed, torsional vibrations of the cantilever are excited by the

Sigrun Hirsekorn is on the research staff of the Fraunhofer Institute for Non-Destructive Testing, D-66123 Saarbrücken. She studied at the University of Braunschweig where she obtained a PhD in theoretical physics.

Ute Rabe studied physics in Heidelberg, Gottingen and Saabrücken and is presently completing her Ph.D. thesis at the University of Saarbrücken.

Walter Arnold heads the Acoustics and Optics Research Group at the Fraunhofer Institute for Non-Destructive Testing and and is a member of the University of Saarbrücken's academic staff. He studied physics at the TU Munich and has worked at the CNRS (Grenoble), IBM (Yorktown Heights), the MaxPlanck Institute (Stuttgart), and Brown Boveri. surface friction force, thus enabling one to image subsurface features with different shear rigidities [5].

We shall demonstrate that an acoustic microscope based on a modified AFM can be operated in the near-field mode to give a lateral resolution on the atomic scale. Moreover, an analysis based on a nonlinear tip-sample force and flexural wave theory for the cantilever predicts materialdependent resonances at lower excitation frequencies. These can, in principle, be used to probe local elastic properties.

\section{A Near-Field AFM}

A commercial AFM was modified [7, 8] to allow the detection of forced ultrasonic cantilever vibrations measured with a high-speed detector to give a direct measurement of vibrations in the sample. The vibrations were generated using an ultrasonic transducer mounted on the sample and excited either by electrical pulses or tone bursts, or by continuous waves with frequency of $1-15 \mathrm{Mhz}$. The modification was necessary because the photodiode in the AFM's built-in beamdeflection sensor used to monitor the laser light beam reflected from the cantilever was too slow to detect ultrasonic vibra-

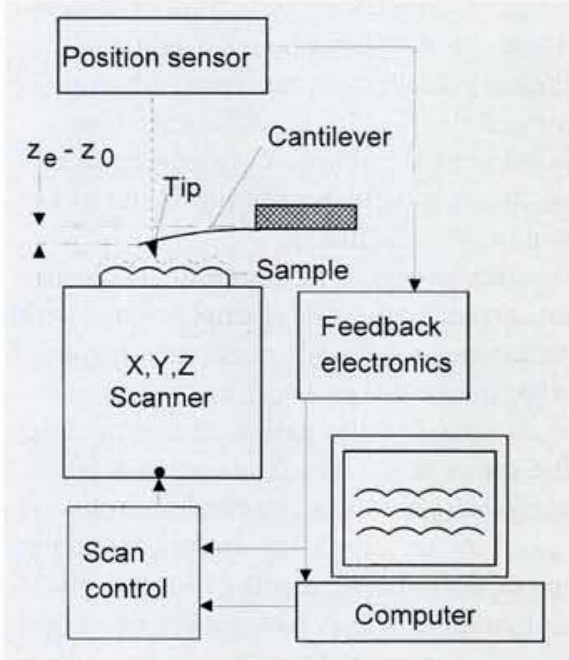

Fig. 1. An atomic force microscope. The sensor is a microfabricated cantilever some 100-200 $\mathrm{lm}$ in length and $1-21 \mathrm{~m}$ in thickness with an integrated tip at its end. During topographic imaging scans, the tip contacts the sample surface or is kept to within about $100 \mathrm{~nm}$. The sample is moved in three directions using a piezoelectric device. A position detector indicates the deflec tion of the cantilever and a feedback circuit connected to its output monitors the cantilever deflection so as to keep a constant tip-sample distance $z$. The preset tipsample distance is $z_{0}$ and the equilibrium distance $z_{e}$.

For ultrasonic imaging, an optical beam-splitter was incorporated to direct one-half of the reflected beam to a high-speed knife-edge detector. The detector's signal was amplified and displayed on an oscilloscope and for imaging, the amplitude of the cantilever vibration was fed into the AFM's second analog input channel.

tions. Our acoustic images effectively record the vibration amplitude of the microscope's cantilever as function of the position on the sample surface.

Fig. 2 gives a comparison between a conventional AFM image and an acoustic

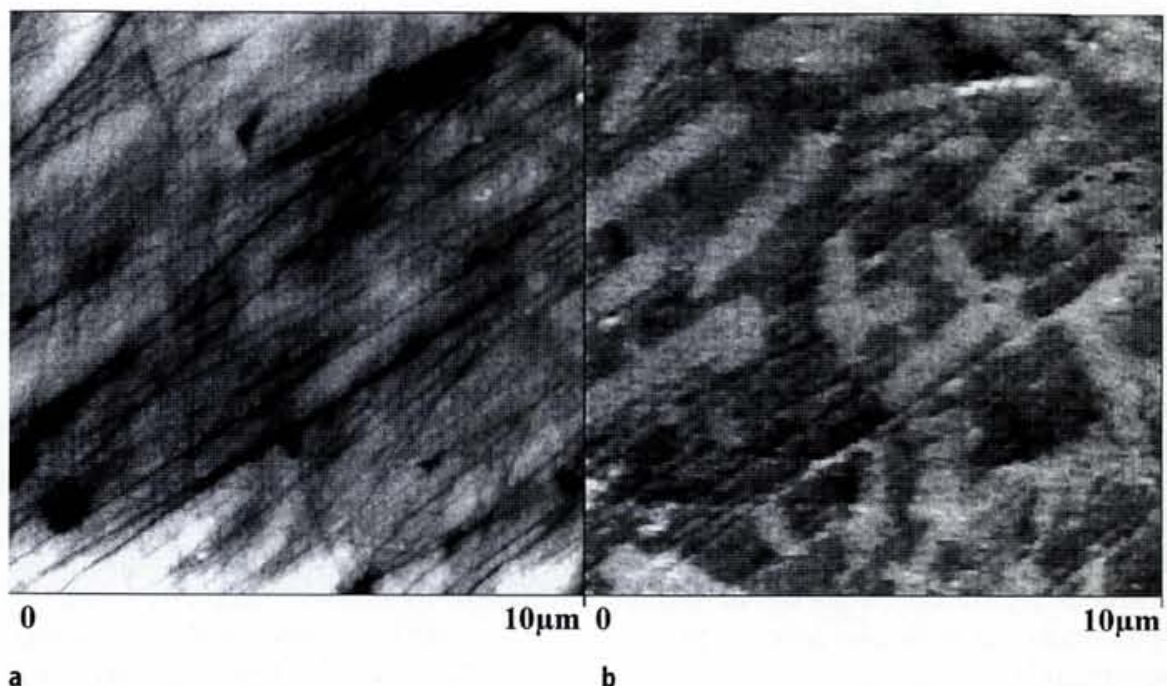


image obtained using our near-field AFM, where an increasing image brightness indicates increasing cantilever vibration amplitude. The SiC fibres in a SiC fibrereinforced alumina composite are resolved in the acoustic image but not in the conventional AFM image.

Fig. 3a gives a topographic image of an extremely flat silica sample coated with tantalum and Figs. $3 \mathrm{~b}$ and $\mathrm{c}$ are ultrasonic AFM images taken at two excitation voltages, with all other parameters remaining the same. The acoustic image taken with the lower excitation amplitude is influenced by the sample topography. A change in contrast observed at the higher excitation amplitude may have been caused by local variations in the adhesion between the tip and the sample.

The important point is that the lateral resolution of these images is comparable to the tip diameter of about $20 \mathrm{~nm}$, which is much smaller than the ultrasonic wavelength. Since the lateral resolution is not determined by the wavelength but by the tip radius, atomic-scale resolution can be reached. This capability to detect ultrasonic waves with an AFM will lead to new applications in ultrasonic imaging.

The amplitude of the cantilever vibrations changes with material properties and inhomogeneities because the transfer of ultrasound from the sample to the cantilever is caused by the material-dependent tip-sample forces. Image contrast in these cases, where forced vibration is induced in the cantilever by the sample, depends on the amplitude of the insonified ultrasonic waves at the sample surface. This is shown in Fig. 4 giving the vibration of the cantilever after sample excitation.

When the sample surface was vibrating sinusoidally with an amplitude of 0.13 $\mathrm{nm}$ (measured using an interferometer), the cantilever followed the surface vibration owing to adhesion between the tip and the surface (Fig. 4a). The amplitude of the cantilever's vibration increased as the surface amplitude increased (Fig. 4b) and the vibrations began to exhibit amplitude modulation. At very large amplitudes (Fig 4c), the cantilever performed complex vibrations of large amplitude. So depending on the magnitude of ultrasonic excitation in the sample, the cantilever could be stimulated to vibrate not only at the insonified frequencies but also at other (lower) frequencies. Fourier transformation of the vibration signals allows the sub-harmonics to be observed in the cantilever's vibration spectrum [9].

By analyzing the tip-sample interac- a)

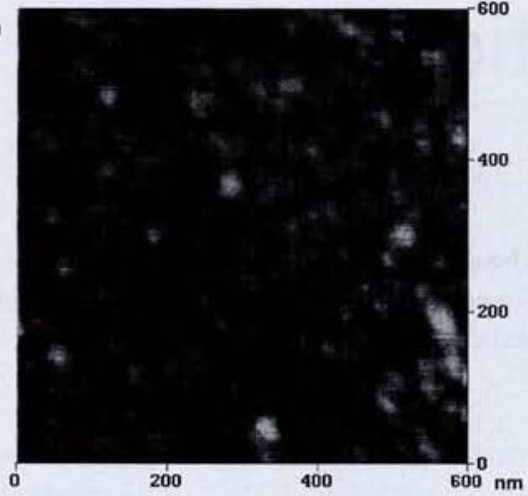

c)

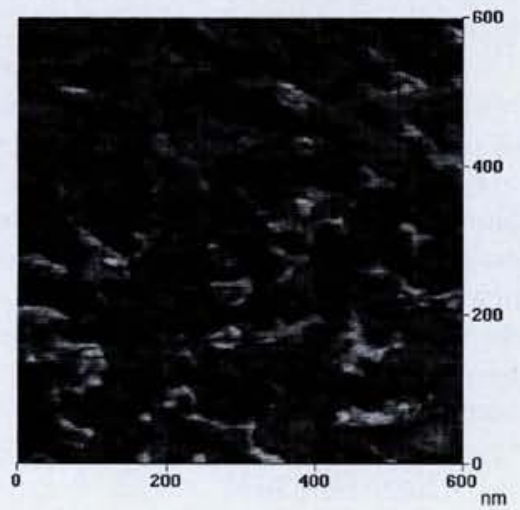

Fig. 4. The measured cantilever vibration forced by ultrasonic waves at the sample surface generated in the sample using an ultrasonic transducer excited with tone bursts of 38 cycles at mean frequency of $5 \mathrm{MHz}$ with amplitudes of $0.13 \mathrm{~nm}(\mathbf{a}), 02.5 \mathrm{~nm}$ (b) and $9.5 \mathrm{~nm}$ (c). In (c), the cantilever vibrates not only at the insonified frequencies but also at lower frequencies. The sample was a $9.5 \mathrm{~mm}$ thick laser mirror made of glass covered with aluminum and a hard protective layer. A standard microfabricated $\mathrm{Si}_{3} \mathrm{~N}_{4}$ cantilever with triangular shape and a spring constant of $0.58 \mathrm{~N} / \mathrm{m}$ was used.

tion, we shall demonstrate that resonance oscillations in the cantilever can, in principle, be used to probe and image local elastic properties.

\section{The Tip-Sample Force}

Compressional waves emitted into the sample by the ultrasonic transducer cause out-of-plane vibrations of the sample's upper surface. Owing to the presence of physical forces acting between the surface and the sensor tip, vibrations are also excited in the cantilever that supports the AFM's sensor tip.

The interpretation and quantitative evaluation of acoustic images recorded using an AFM therefore requires investigation of the interaction force [10] between the sensor tip and the surface of the sam- b)

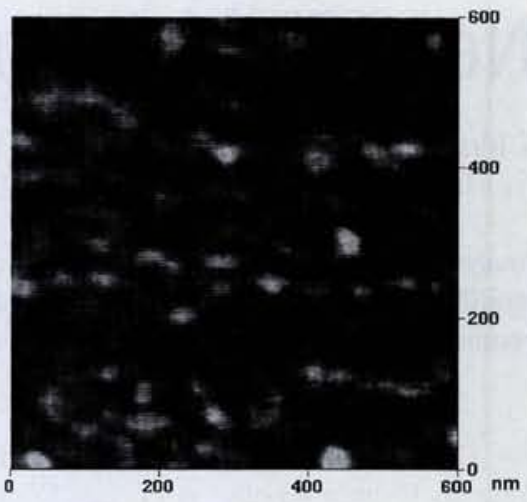

Fig. 3. A silica glass sample coated with a tantalum thin film. A conventional AFM topographic image (a) compared with acoustic AFM images taken using an ultrasonic transducer excitation voltage of $0.8 \mathrm{~V} \mathrm{(b)}$ and $2.5 \mathrm{~V}(\mathbf{c})$, with all other parameters such as excitation frequency (1.91 $\mathrm{MHz})$, repetition rate $(10 \mathrm{kHz})$, and number of cycles in the tone burst $(50)$ remaining constant. The sample is very smooth since the grey scale covers a height of only $3.9 \mathrm{~nm}$.

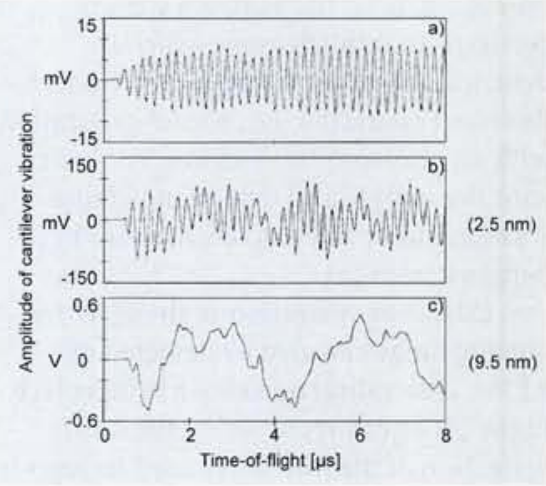

ple to predict the reaction of the cantilever to the ultrasonic waves in the sample. However, the tip-sample force cannot be measured directly as function of the tipsample separation distance $z$ because the distance is determined by the length of the cantilever. So a force calibration curve must recorded before scanning.

Fig. 5 shows a force calibration curve obtained using a silicon beam cantilever approaching a chromium surface. The sample was moved slowly perpendicular to its surface while the cantilever deflection was recorded as a function of $z$. The cantilever's deflection and the tip-sample interaction were in force equilibrium. As the cantilever approached the sample it reached a point where the tip jumped onto the sample surface and the force gradient 
became larger than the cantilever's spring constant. Further approach caused a decrease of the cantilever deflection to zero, followed by deflection in the other direction when the tip was pressed into the sample and a Hertzian contact was formed. On pulling the cantilever away from the sample, the cantilever deflection went through the zero point and then jumped at a separation distance different to the one for the approach.

Fig. 6 gives the force curve calculated on the basis of the calibration curve and a simple model to describe the forces between the sensor tip and the sample surface. According to the model, the most important force in the contact mode when the tip is in contact with the sample is the contact deformation force $F_{\text {cont }}(z)$ arising when an elastic sphere is pressed into an elastic surface (Hertzian contact), with a correction term for adhesion.

If the AFM's tip is not maintained in a vacuum, the forces operating in the socalled attractive mode when the tip is slightly removed from the sample surface are mainly determined by the adhesive force $F_{W F}$ between water films covering the tip and sample. Generally speaking, no single force dominates in the intermediate region between the contact and attractive modes. This suggests that one can describe the force $F_{\text {int }}$ in the intermediate regime from the connection point $z_{\mathrm{a}}$ determined by continuity conditions to $2 d$, the separation distance at which the water film breaks, by an exponential interpolation between the contact and attractive forces. Hence, the tip-sample force $F_{s}(z)$ is given by:

$$
\begin{aligned}
F_{s}(z) & =F_{\text {cont }}(z) & & \text { for } 0 \geq z \\
& =F_{\text {int }}(z) & & \text { for } z_{\mathrm{a}} \geq z \geq 0 \\
& =F_{\mathrm{WF}}(z) & & \text { for } 2 d \geq z \geq z_{\mathrm{a}} \\
& =0 & & \text { for } z>2 d .
\end{aligned}
$$

The forces are chosen to be continuous except at $z=0$ and at $z=2 d$ given by the jump point on approaching the tip to the sample when water films of thickness $d$ on the sample and tip come into contact. The gradient of the contact force increases rapidly within a very small region to infinity at $z=0$ so numerical estimation of a connection point between $F_{\text {cont }}$ and $F_{\text {int }}$ that gives a continuous slope needs careful analysis.

Standard expressions for $F_{\text {cont }}$ and $F_{\text {WF}}$, known materials constants, and an exponential interpolation of $F_{s}(z)$ between the connection and separation points yielded the force curve plotted in Fig. 6.

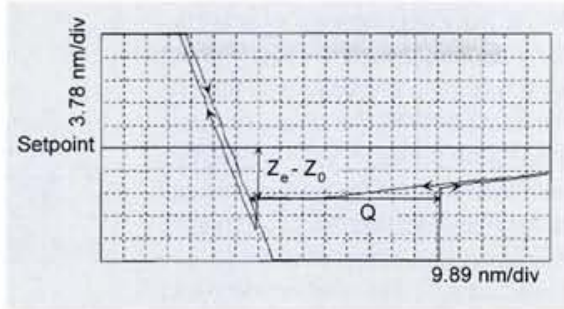

Fig. 5. A force calibration curve for a silicon tip on a chromium surface. The vertical axis corresponds to the tip-sample force and the tip-sample separation z increases from left to right. The difference between the separations at which the tip jumps to and detaches from the surface on approaching and moving away is $Q$ and $z_{0}$ and $z_{\mathrm{e}}$ are the set and equilibrium separations.

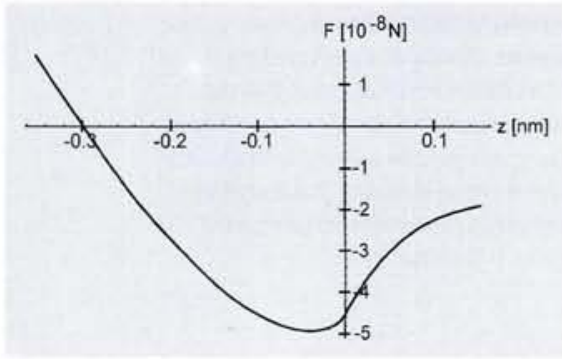

Fig. 6. The tip-sample force $F_{s}(z)$ as a function of $z$ calculated using the force calibration curve of Fig. 5 and a model for $F_{s}(z)$.

the tip adopts a new equilibrium position $\bar{z}_{\mathrm{e}}$ which obeys

$$
k_{\mathrm{c}}\left(\bar{z}_{\mathrm{e}}-z_{\mathrm{o}}\right)=(1 / T) \int_{0}^{T} \mathrm{~d} t \cdot F_{\mathrm{S}}[(z(t)-a(z)]
$$

where $T$ is the period of the tip and sample vibrations. The ultrasound in the sample causes a change in the mean cantilever deflection. If the vibration amplitudes of the sample surface and the cantilever are small a linear approximation can be used for the force $F_{\mathrm{S}}(z)$. Assuming that the sensor tip remains at the same position, i.e., $\bar{z}_{\mathrm{e}}=z_{\mathrm{e}}$, the time average of Eq. (2) yields Eq. (1). So a change in the mean cantilever deflection originates from the fact that the tip-sample force is non-linear.

The cantilever deflection calculated using Eq. 2 assuming that the cantilever is forced to vibrate at the single frequency $\omega_{\mathrm{u}}$ is plotted in Fig. 7 for two different assumptions about the forced cantilever vibration. It is seen that the calculated values for no transfer of ultrasound into the cantilever (dashed line) are much lower than the measured effects.

Convincing agreement with the The surface position as a function of time $t$ is given by $a(t)=a_{0} \sin \omega_{\mathrm{u}} t$ on exciting the sample surface with an ultrasonic vibration of amplitude $a_{\mathrm{o}}$ and frequency $\omega_{\mathrm{u}}$. The distance $z$ between the sensor tip and the sample surface is modulated and experimental data is obtained if it is assumed that the cantilever oscillations change phase with respect to the sample oscillations on increasing the ultrasonic amplitude in the sample (solid line in Fig.

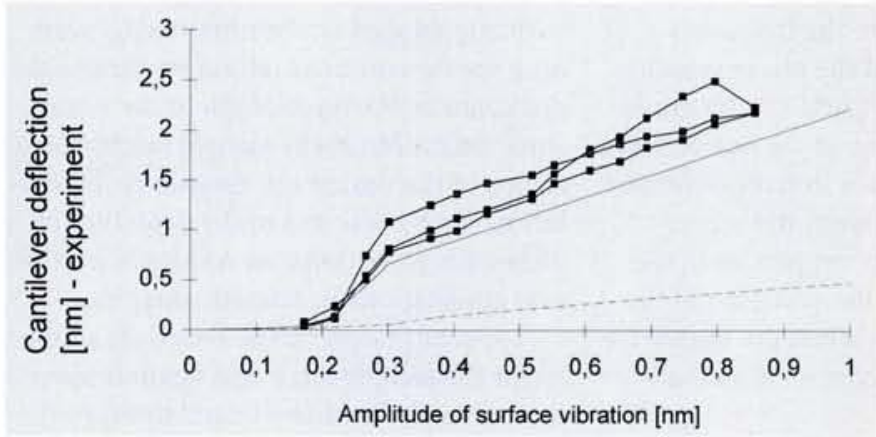

Fig. 7. The change in cantilever deflection resulting from ultrasonic vibrations at the surface of the sample. The measurements do not agree with the theoretically predicted curve (dashed line) obtained assuming that the phase between the sample and cantilever vibrations does not change. Agreement is achieved if the phase of the cantilever vibrations is allowed to change (the solid line was calculated by interpolating between a zero phase shift at surface vibration amplitudes $a_{0}$ below $0.2 \mathrm{~nm}$ and a $180^{\circ}$ phase shift for amplitudes between $0.3 \mathrm{~nm}$ and $1.0 \mathrm{~nm}$ ). 
7). This change of phase is understandable because of the low cantilever spring constant: the cantilever is unable to follow the the movement of the sample's surface at large vibration amplitudes.

\section{Resonances}

For sufficiently high excitation amplitudes in the sample one needs to consider the dynamics of the cantilever. For cantilever deflection perpendicular to the sample surface, flexural vibrations are dominant, which, if damping is neglected, obey the differential equation

$$
E I \partial^{4} y / \partial x^{4}=-\rho A \partial^{2} y / \partial t^{2}
$$

where $x$ is the coordinate in the longitudinal direction, $y(x, t)$ is the deflection of a beam with elastic modulus $E$ and density $\rho$, and for a rectangular beam of width $w$ and thickness $b$ the area moment of inertia $I$ is $w b^{3} / 12$. The solutions of this differential equation are real or imaginary exponential functions which lead to a dispersion relation that depends on the boundary conditions for deflection of the beam.

One end of the AFM cantilever beam of length $L$ is clamped and the free end is in contact with the sample surface. The tip-surface force $F_{s}(z)$ is entered into the boundary conditions by restricting ultrasonic stimulation to very small amplitudes so that a linear approximation is valid. The force given by the contact mode force $F_{\text {cont }}$ can then be replaced by its expansion around the equilibrium position up to the first-order term. The characteristic equation for the beam now becomes

$$
\begin{aligned}
& \sinh \left(k_{n} L\right) \cos \left(k_{n} L\right)-\cosh \left(k_{n} L\right) \sin \left(k_{n} L\right) \\
& =\left(k_{n} L\right)^{3} k_{c} / 3 k^{*}\left[1+\cosh \left(k_{n} L\right) \cos \left(k_{n} L\right)\right]
\end{aligned}
$$

with the cantilever spring constant given by $k_{\mathrm{c}}=E a(b / L)^{3 / 4}$ and $k^{*}=-\mathrm{d} F_{s}(z) / \mathrm{d} z$ at $z$ equal to $z_{\mathrm{e}}$ The wave number $k_{n}$ of the $n$th component is related to the frequency $f_{n}$ of waves in the beam and the phase velocity $v_{\text {ph }}$ by $k_{n}=2 \pi f_{n} / v_{\text {ph }}$. Because $k^{\star}$, the gradient of the force function at the equilibrium point, involves materials' constants for the sample, the equation opens the way to determining the elastic properties of the sample by measuring the variation of the resonance frequencies when the sensor tip of the cantilever is in contact with the sample surface.

This eigenfrequency equation for the three lowest resonances for a clamped cantilever beam with the free end in contact with the sample surface is plotted in Fig. 8 as a function of the so-called contact stiff-
Fig. 8. This eigenfrequency equation for the three lowest resonances $(n=1-3)$ for a clamped cantilever beam with the free end in contact with the sample surface. The angular frequency $\omega_{n}=2 \pi f_{n}$ of resonance vibrations in the beam normalized to the zero-order frequency $\omega_{0}$ is plotted (solid points) as a function of the socalled contact stiffness ( $k^{*}$ normalized to $k_{f}$ ). The shaded areas indicate gaps in the vibration spectra where there are no resonances. The open points are for a pointmass model which assumes that the mass of the cantilever is concentrated in one point fixed by a spring with the cantilever spring constant. This model does not predict resonance frequencies of order higher than 1.

Fig. 9. Experimentally determined displacement of the first three resonance frequencies of an AFM tip in contact with chromium and glass surfaces. The amplitude of the cantilever vibration is plotted as a function of the frequency. The dashed lines give the calculated positions of the resonance frequencies in the case that the tip does not contact the sample.

ness $\left(k^{*}\right.$ normalized to $k_{\mathrm{c}}$ ). Gaps in the vibration spectra of the cantilever motion are indicated by the shaded areas. Fig. 9 indicates that these gaps show up in data giving the experimentally determined displacement of the first three resonance frequencies of our near-field AFM cantilever in contact with chromium and glass surfaces. It can be seen that the displacement of the resonances depends upon the type of sample, thus confirming that the local elastic properties of the sample can be measured and in principle used to obtain high-resolution acoustic images

\section{Perspectives}

An atomic force acoustic microscope operated in the near-field mode where the sensor tip is in physical contact with the sample surface allows one to determine material constants at the sample's surface. Acoustic images can be obtained by scanning the tip and their lateral resolution is not given by the wavelength of the ultrasonic excitation in the sample but by the radius of the sensor tip. Atomic-scale resolution can be reached so the detection of ultrasonic waves with an AFM will lead to new applications in acoustic imaging.

A quantitative model based on a nonlinear tip-sample force and flexural wave theory for the cantilever used to support the sensor tip in a near-field AFM shows that the surface stiffness of a specimen can be derived from the shift of the resonance frequencies at low excitation amplitudes. This opens the way forward to high-

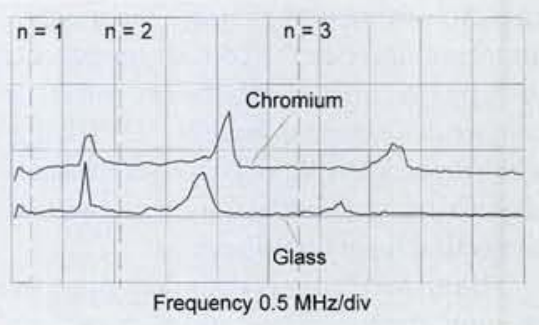

resolution acoustic imaging and to determining the local elastic properties of the sample by measuring the displacement of the resonances.

A thorough analysis of the nonlinear transfer of mechanical oscillations in an AFM within the $\mathrm{MHz}$ frequency range far above the frequency of the lowest resonance may be able to provide information on the all-important local adhesion between the tip and the sample.

\section{References}

[1] B. Cretin \& F. Sthal, Appl. Phys. Lett. 62 (1993) 829.

[2] P. Vairac, F. Sthal \& B. Cretin in Proc. IEEE Ultrasonics Symp.; Eds: M. Levy, S.C. Schneider \& B.R. McAvoy (Inst. Electrical \& Electr. Eng., 1994) 1401.

[3] W. Rohrbeck \& E. Chilla, Phys. stat. sol. (a), 131 (1992) 69.

[4] O. Kolosov \& K. Yamanaka, Jpn. J. Appl. Phys. 32 (1993) 22.

[5] K. Yamanaka, H. Ogiso \& O. Kolosov, Appl. Phys. Lett. 64 (1994) 178.

[6] E. Chilla, T. Hesjedahl \& H.-J. Frohlich in Proc. IEEE Ultrasonics Symp., Eds: M. Levy, S.C. Schneider \& B.R. McAvoy (IEEE, 1994) 363.

[7] U. Rabe, M. Dvorak \& W. Arnold, Thin Solid Films 264 (1995) 165.

[8] U. Rabe \& W. Arnold, Appl. Phys. Lett. 64 (1994) 1493; U. Rabe \& W. Arnold, Ann. der Physik 3 (1994) 589; U. Rabe, K. Janser \& W. Arnold, Proc. 22nd Int. Symp. Acoustic Imaging (Florence, 1995) to be published.

[9] N.A. Burnham et al., Phys. Rev. Lett. 72 (1995) 5092.

[10] N.A. Burnham \& R.J. Colton, J. Vac. Sci. Technol. A7 (1989) 2906; N.A. Burnham et al., Nanotechnology 4 (1993) 64. 